\title{
Sag, Swell and Interruption Detection Using Wavelet in LabVIEW
}

\author{
D. P. Mishra
}

\begin{abstract}
This paper presents that for monitoring power quality problems the wavelet transform is a powerful tool. Different wavelet analysis (discrete or wavelet-packet transform), with different mother wavelet, decomposition tree and different sampling rate is performed on the input signal in real-time. The wavelet transform and the proposed hardware and software solutions adopted for setting up the instrument are presented. The real signals from chroma programming are used in LabVIEW algorithm by Data Acquisition (DAQ) card. To obtain the results DAQ digitizes the input line signal. Very accurately and satisfactory voltage sag, swell, interrupt are detected in the test results. It demonstrates the good performance of the instrument developed for the detection and analysis of different power quality disturbances.
\end{abstract}

Index Terms - Chroma programming, power quality, virtual instruments, wavelet transforms.

\section{INTRODUCTION}

Now a days the manufacturing industry face problems due to the distortion of the electrical supply. All kinds of transducers, PLC apparatuses and automatic products based on computers are sensitive to small distortion of supply voltage. Interruption affects the production of the manufacturing industry. The result is very expensive loss. More attention has been given in the power quality problems. [1] Since power quality distortion has harmful effects on the electric power system, there is a need to analyze the disturbances by detection and localization. Frequency components of a periodic and stationary voltage or current waveform are known by discrete Fourier transform (DFT). Frequency domain information obtained by DFT analysis only depends on the time window width. The time information of the signal is not obtained. [2]

In power quality it is important to know the time information rather than the exact frequency of a disturbance.

Wavelet transform can satisfy this type of analysis. Wavelet of Daubechies (4db) is applied to detect voltage sag, voltage swell, and voltage interrupt. The proposed algorithm is simple and proves to be accurate when applied to different power quality distortion level.

\section{WAVELET THEORY}

FFT analysis only provides information in the frequency domain with a resolution that depends on the time window width. No time information about the signal is provided. It is

Manuscript received December 13, 2012; revised March 11, 2013.

D. P. Mishra is with the Department of Electrical Engineering, IIIT Bhubaneswar, Odisha, India (e-mail: debani@iiit-bh.ac.in). very difficult for the analysis of electrical power quality disturbances. In order to overcome this limitation the Windowed Fourier Transform (WFT) is used to provide frequency-time spectrum. The Windowed Fourier Transform (WFT) is also called Short Time Fourier Transform (STFT). But STFT has the limitation of fixed window size. Window resizing flexibility cannot be achieved in the STFT which greatly affects both frequency and time resolution. To overcome the resolution problem the continuous wavelet transform is developed as an alternative approach to the short time Fourier transform. Wavelet Transform (WT) method provides frequency-time spectrum along with flexible resizing of the window, therefore preserving time and frequency information. It is especially suited to analysis of non-stationary signals. So wavelet allows the simultaneous evaluation of a signal in the time and frequency domains with different resolutions [3]-[5]. In Wavelet analysis, Discrete Wavelet Transform (DWT) provides different sized frequency bands and less tree branches. Therefore less computational time is required. On the other hand, Wavelet Packet Transform (WPT) is considered as the generalization of the wavelet decomposition. It provides the full tree of decomposition for the analyzed signal. Although it provides uniform frequency bands or levels, it requires a large amount of calculations [6].

Wavelet and Fourier transforms represent a signal through a linear combination of their basis functions. The basis functions are dilations of cosine and sine signals (each spanning the entire time interval) for Fourier transforms. For wavelet transforms the basis functions are translations and dilations of function. The function is termed as Mother Wavelet along with a scaling function (each spanning a logarithmically reduced subinterval). The dilations of both sets of basis functions are possible because of their frequency localization. So it allows us to obtain frequency information. But the most important difference between the two sets of basis functions is time localization. The basis functions of wavelet transform are compact, or finite in time. This feature allows the wavelet transform to obtain time information as well as frequency information [7].

\section{AlgORITHMS OF WPT}

The wavelet-packet transform (WPT) can be used to obtain a uniform frequency decomposition of the input signal. In the WPT both the output low-pass and high-pass filters (the detail and the approximation coefficients) are decomposed to produce new coefficients. Fig. 1 shows the two-level wavelet decomposition tree. The WPT has the same frequency bandwidths in each resolution but DWT does 
not have this property. It does not increase or lose the information during the mode of decomposition. So the signal contains the original information. Therefore, the signal with great quantity of middle and high frequency components can offer superior time-frequency analysis. For non stationary signals WPT is a suitable signal processing tool. Unlike high and low frequencies, the same frequency bandwidths can provide good resolution.

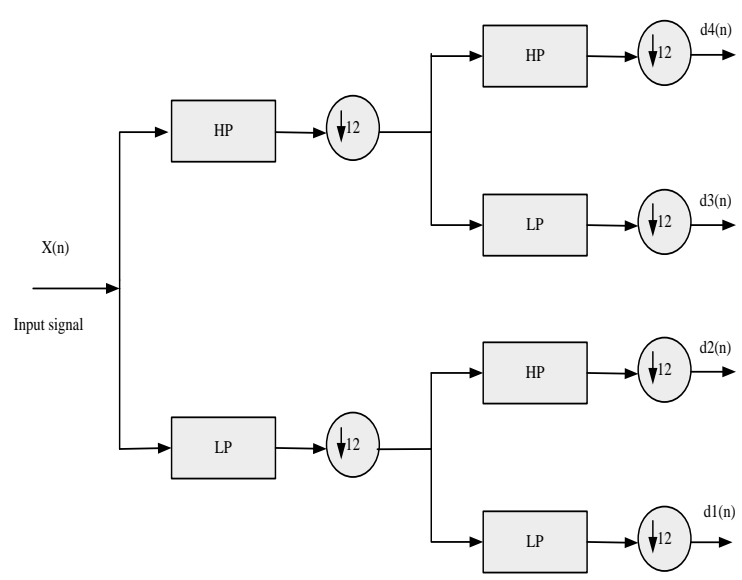

Fig. 1. Two-level wavelet decomposition tree for WPT analysis

Fig. 2 shows the uniform frequency decomposition of the input signal. According to IEC standard 61000-4-7 the wavelet-packet transform is used instead of DWT for selecting the sampling frequency and the wavelet decomposition tree. The uniform output frequency bands can be selected for corresponding frequency bands of the input signal [8].

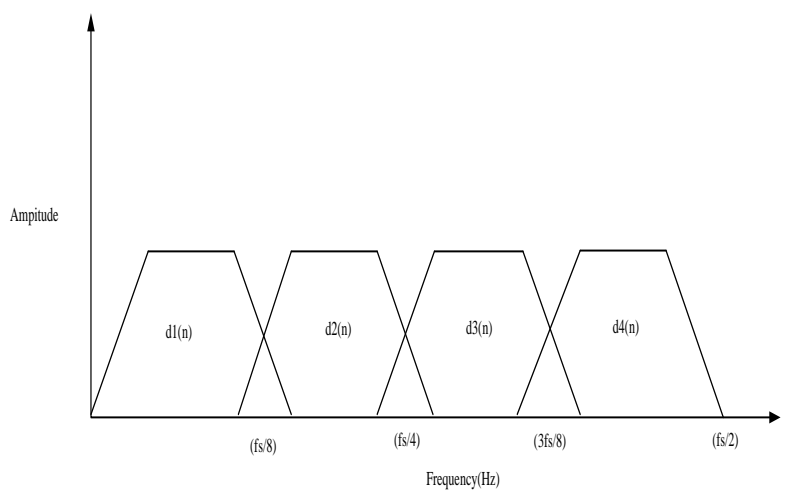

Fig. 2. Output frequency bands of the wavelet decomposition tree

In Fig. 3, $(i, j)$ refers to the node, where $i$ is the level number and $j$ is the number of node in certain level. The original signal represents $(0,0)$ in the frequency band $\left(0, f_{s}\right)$. $f_{s}$ is the sample frequency of original signal. $(1,0)$ represent the signal in the $\left(0, f_{s} / 2\right)$ frequency band; $(1,1)$ represent the signal in the $\left(f_{s} / 2, f_{s}\right)$ frequency band; $(2,0)$ represent the signal in the $\left(0, f_{s} / 4\right)$ frequency band; $(2,1)$ represent the signal in the $\left(f_{s} / 4, f_{s} / 2\right)$ frequency band, and so on. So level $i$ wavelet packet decomposes the original signal into $2 j$ frequency band.

So the Wavelet Packet Transform is accurate and gives values which are closer to the true values and is more suitable for distortions and non-linear loads. It also provides uniform frequency sub-bands which are an important advantage.

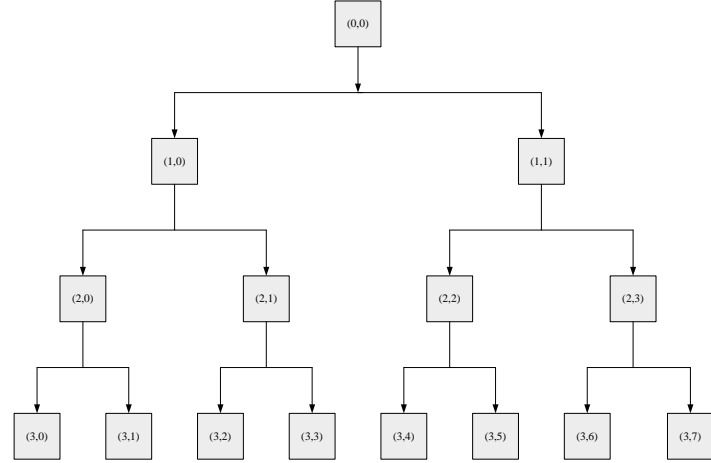

Fig. 3. The tower structure of wavelet package decomposition

\section{Test Results by Using Chroma Programming AND LABVIEW}

Continuous monitoring was carried out at the power system core lab of Indian Institute of Technology, Delhi. Fig. 4 shows Lab-PC, DAQ board, full Specifications, I/O Connector , cable and demo-box with terminals. Demo-box contains 6 analog inputs from A0-A5, 4 digital outputs from D0-D3, and a Ground terminal. Model 8112 clamp adaptor current probe or voltage probe real time signal data were collected from Chroma 615XX Series programme and given to analog input terminal of the Demo-box. From Demo-box the signal was sent to LabVIEW 8.6 algorithm by Data Acquisition hardware. Results were displayed on front panels of the computer screen in LabVIEW software. Single phase voltages and currents were continuously sampled at a sampling rate of $3.2 \mathrm{KHz}$, which correspond to 64 samples per cycle with $50 \mathrm{~Hz}$ fundamental frequency. The proposed monitoring technique detected and localized all the transient disturbances in the distorted signal using db4 (Daubechies) level 4 decomposition. The information about the position and duration of a power system disturbance was obtained from the detail coefficients $\mathrm{d} 1$. When disturbance exists the signal coefficient $\mathrm{d} 1 \mathrm{(n)}$ is non-zero.

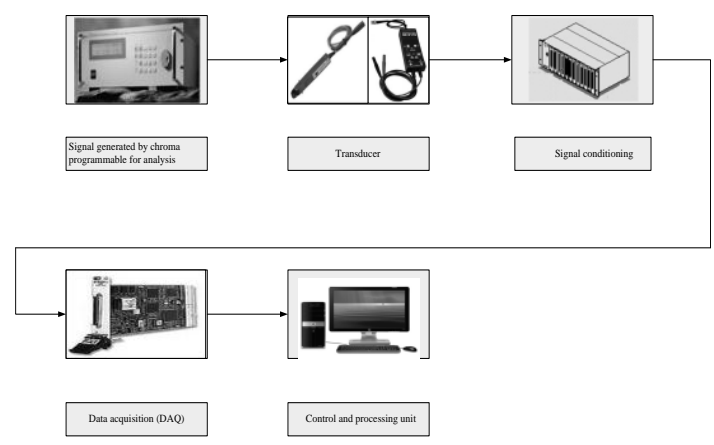

Fig. 4. Hardware architecture of the Chroma and LabVIEW

\section{RESULTS}

\section{A. Detection of Pure Sine Wave Using Wavelet Packet Transform in Real Time}

Fig. 5 shows the pure sine wave generated in chroma. Fig. 6 shows the pure sine wave signal in the front panel of LabVIEW. The horizontal axis presents the time in seconds and the vertical axis presents the magnitude. Fig. 7 shows the db4 (Daubechies) level 4 decomposition levels. The 
horizontal axis presents the time and the vertical axis presents the magnitude of the resolution levels.

In this case, the resolution levels (Fig. 7) do not detect any changes. It will appear as a dotted line in the $\mathrm{db} 4$ (Daubechies) level 4 decomposition levels. The magnitude of the resolution levels is zero. The pure sine wave will be used as a reference in comparison with disturbed power signal cases.

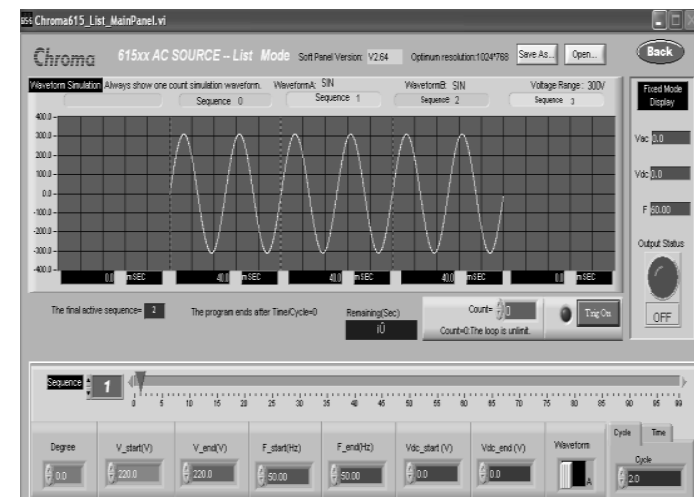

Fig. 5. Pure sine wave form generation in chroma programming

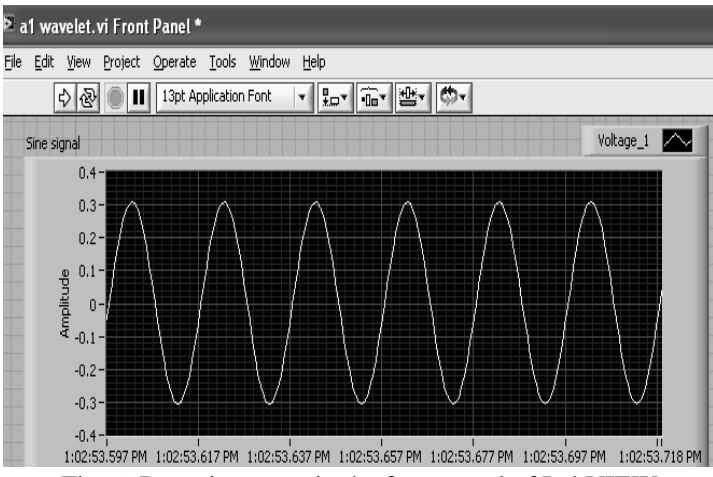

Fig. 6. Pure sine wave in the front panel of LabVIEW

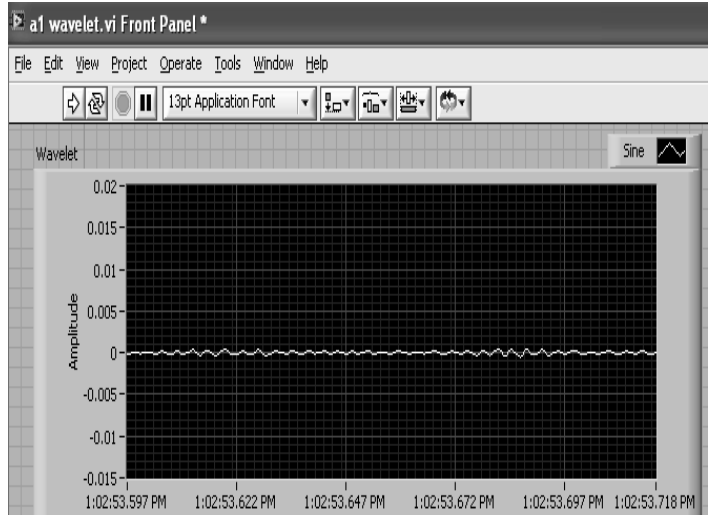

Fig. 7. Detection and localization of pure sine wave using WPT

\section{B. Detection of Voltage Interruption Using Wavelet Packet Transform in Real Time}

Circuit breaker tripping, faults in power system, equipment failures and control malfunctions cause interruption in power network. So during Voltage interrupt, magnitude of the voltage decreases to 0.0 p.u of the rated value for duration of time greater than 1 minute and then resumes the normal value based on IEEE standard 1159 [9]. Voltage interrupt signal is generated in chroma programming (Fig.8) and simulated in LabVIEW G programming as shown in Fig. 9. The wave form interrupts from 5:35:25:766 to
5:30:25:807. The sampling frequency and supply frequency are $3.2 \mathrm{KHz}$ and $50 \mathrm{~Hz}$ respectively. The coefficients of details at level 4 are shown in Fig. 10. A spike is seen at the starting and end point of the interrupt in WPT at times from $5: 35: 25: 766$ to $5: 30: 25: 807$, from which, we can obtain the beginning time and the ending time of voltage interrupt accurately.

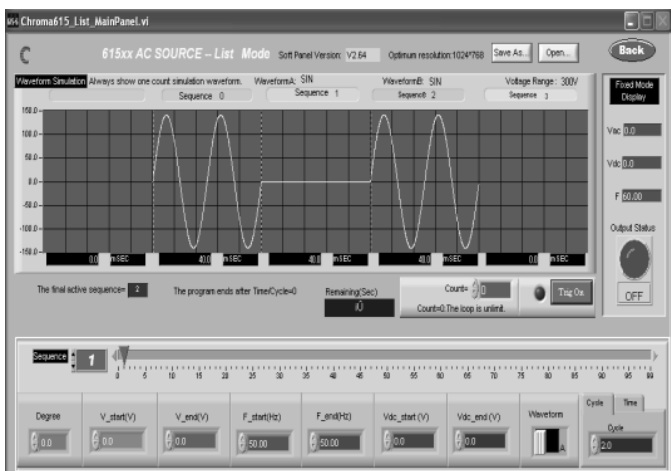

Fig. 8. Voltage interrupt signal generated in chroma programming

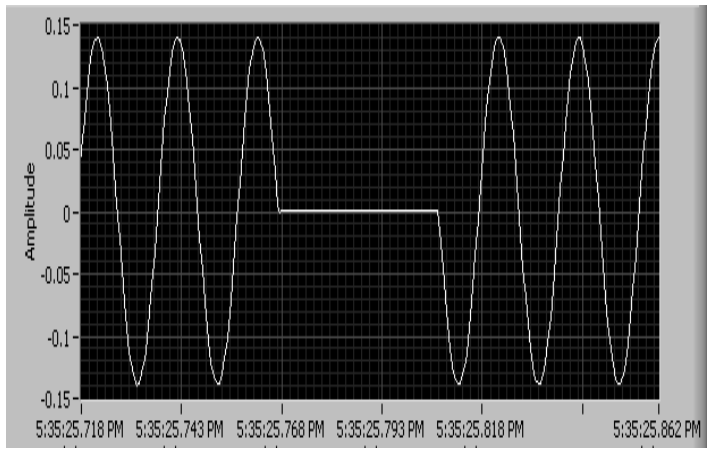

Fig. 9. Voltage interrupt signal shown in the front panel of LabVIEW

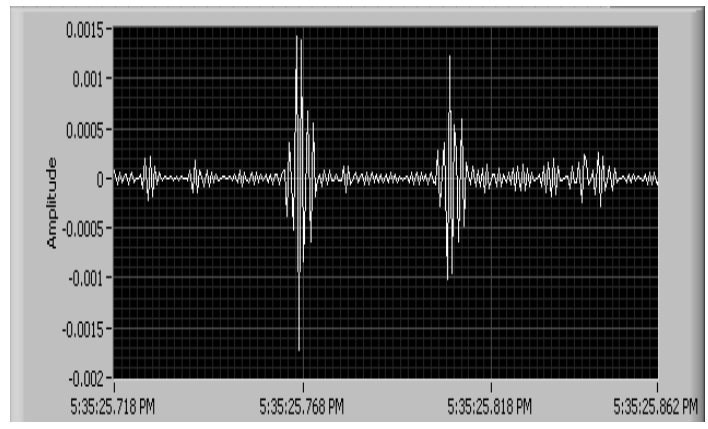

Fig. 10. Detection and localization of interrupt wave using WPT

\section{Detection of Voltage Swell Using Wavelet Packet Transform in Real Time}

Load switching, capacitor switching and system voltage regulation cause swell formation in a pure sine wave. During voltage swell magnitude increases from 1.1 to 1.8 p.u of the rated value in the time duration of 0.5 to 30 cycles continuously, and then resumes the normal value.

Fig. 11 shows the generation of swell signal in chroma programming. The swell magnitude is increased to $220 \mathrm{~V}$ over an interval of 2 cycles. Then it resumes to normal sine wave magnitude of 150V. As shown in Fig.12, the signal of voltage swell is simulated in LabVIEW G programming. The wave form contains disturbance of swell from time 
5:27:39:934 to 5:27:39:974. The sampling frequency and supply frequency are $3.2 \mathrm{KHz}$ and $50 \mathrm{~Hz}$ respectively. It is assumed that the lifting wavelet of Daubechies (db4) is decomposed to level 4 . The coefficients of details at level 4 are shown in Fig. 13. A spike is seen at the starting and end point of the swell in WPT from time 5:27:39:934 to $5: 27: 39: 974$, from which, we can obtain the beginning time and the ending time of voltage swell accurately.

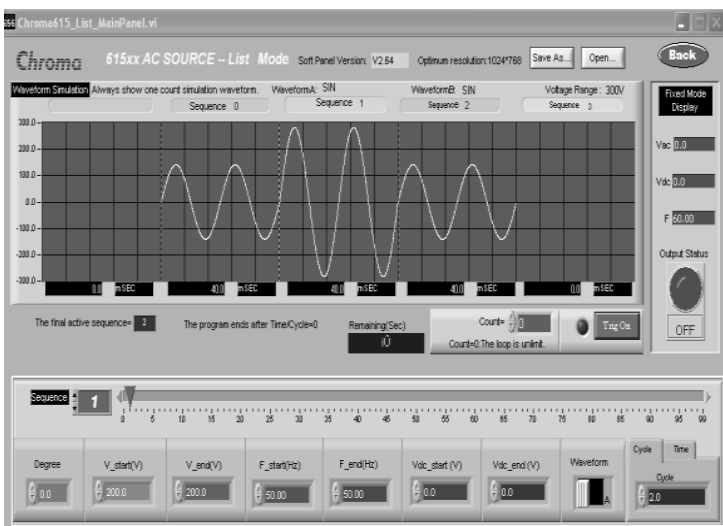

Fig. 11. Swell signal generation in chroma programming

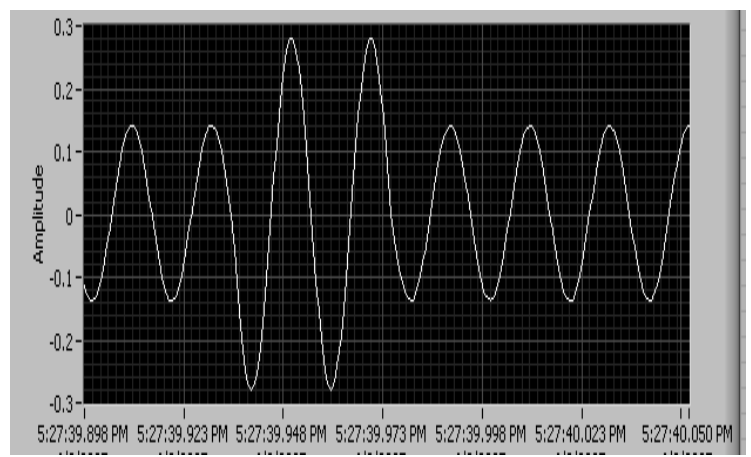

Fig. 12. Swell voltage signal shown in the front panel of LabVIEW

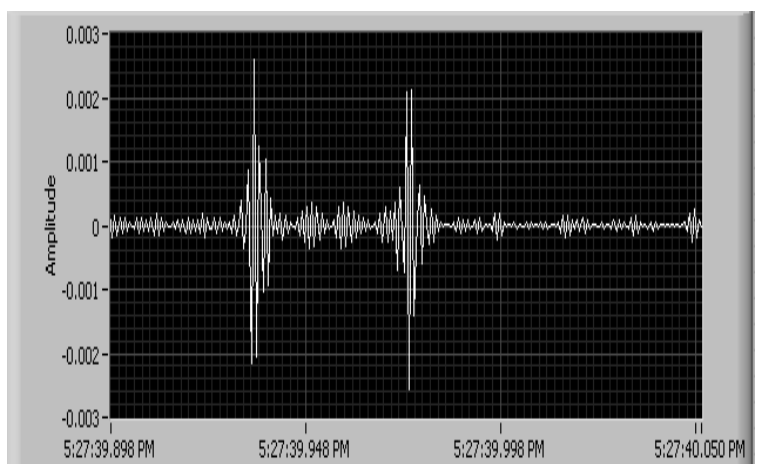

Fig. 13. Detection and localization of swell in pure sine wave using WPT

\section{Detection of Voltage Sag Using Wavelet Packet Transform in Real Time}

Fig. 14 shows generation of sag disturbances in chroma programming. The voltage decreases to $120 \mathrm{~V}$ for 2 cycles and then resumes the normal sine wave voltage of $220 \mathrm{~V}$. The wave form contains disturbance of sag from time 5:59:35:721 to 5:30:00:768. The signal of voltage sag is simulated in LabVIEW $G$ programming as shown in Fig. 15. The sampling frequency and supply frequency are $3.2 \mathrm{KHz}$ and $50 \mathrm{~Hz}$ respectively. It is assumed that the lifting wavelet of Daubechies (db4) is decomposed to level 4. The coefficients of details at level 4 are shown in Fig. 16. A spike is seen at the starting and end point of the sag in WPT from time 5:59:35:721 to 5:30:00:768, from which, we can obtain the beginning time and the ending time of voltage sag accurately.

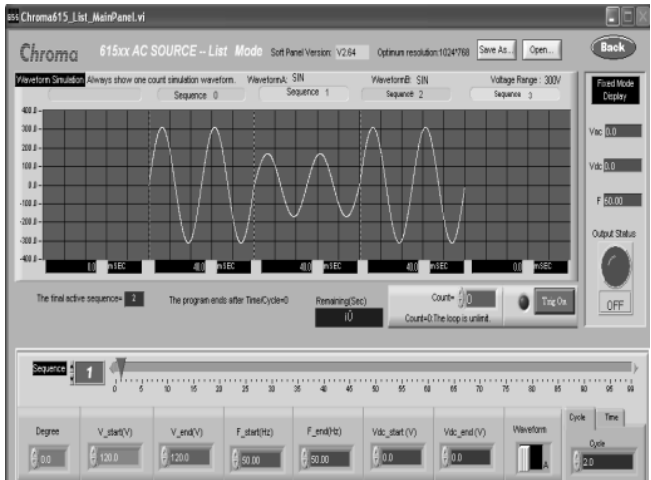

Fig. 14. Waveform generation in chroma programming with sag disturbances

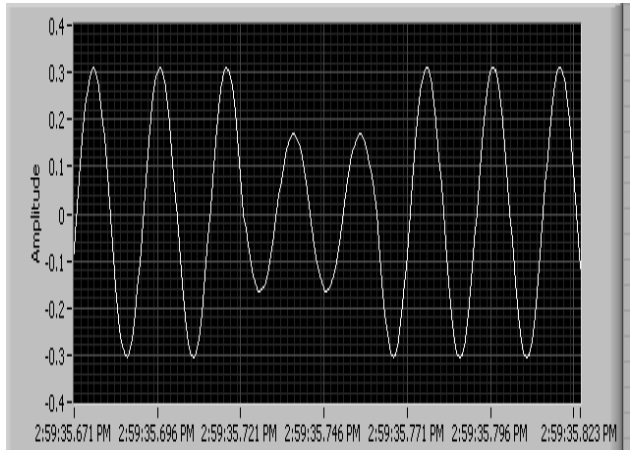

Fig. 15. Sag voltage signal shown in the front panel of LabVIEW

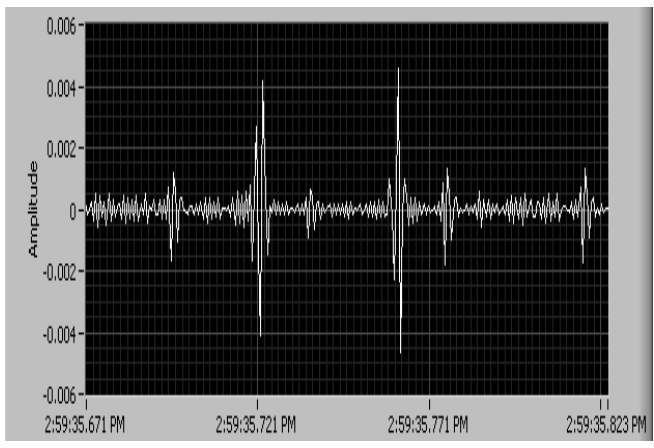

Fig. 16. Detection and localization of sag by using WPT

\section{CONCLUSION}

By choosing the most suitable wavelet family and mother wavelet, and then applying the Wavelet packet transform the problem of spectral leakage can be minimized when analyzing power system disturbances. The main advantage of the proposed method is the separation of power quality problems that overlap in both time and frequency. Now a day's virtual measurement instrument is very good instrument for detection and analysis of power quality disturbances in a low voltage distribution system using wavelets. Wavelet packet transform is the faster transform for detection and compression purpose in non-stationary signal to identify the origins of power quality problems by utilizing power quality data, especially voltage signal. So it helps to improve the reliability of power systems. This may improve the computational efficiency of the system. It has the 
advantage of finding the duration of disturbances in real time operation with low cost implementation.

The magnitudes of the spikes are different and are not symmetrical as shown in Fig.10 and Fig.13. So it only determines the occurrence of sag and swell but it cannot give direct information about the magnitude of sag.

\section{REFERENCES}

[1] J. Douglas, "Solving problems of power quality," EPRI journal, vol. 18, no. 8, pp. 6-15, Dec. 1993.

[2] General guide on harmonics and Interharmonics measurement and instrumentation, for power supply systems and equipment connected thereto, International Electro technical Commission, IEC 61000-4-7, Electromagnetic compatibility (EMC), Part 4-7: Testing and measurement techniques, Switzerland, 2002.

[3] S. Santoso, E. J. Powers, W. M. Grady, and P. Hofmann, "Power Quality assessment via wavelet transform analysis," IEEE Trans. Power Delivery, vol. 11, no. 2, pp. 924-930, Apr. 1996.

[4] L. Angrisani, P. Daponte, M. D'Apuzzo, and A. Tesla, "A measurement method based on the wavelet transform for power quality analysis," IEEE Trans. Power Delivery, vol. 13, no. 4, pp. 990-998, Oct. 1998.
[5] W. K. Yoon and M. J. Devaney, "Power measurement based on the wavelet transform," IEEE Trans. Instrum. Meas., vol. 47, pp. 1205-1210, Oct. 1998

[6] A. Domijan, A. Hari, and T. Lin, "On the selection of appropriate filter bank for power quality monitoring," Int. J. Power Energy Syst., vol. 24, pp. 46-50, 2004.

[7] G. Strang, "Wavelet Transforms vs. Fourier Transforms," Bulletin (New Series) of AMS, vol. 28, no. 2, pp. 288-3051993.

[8] J. Barros and R. I. Diego, "A new method for measurement of harmonic groups in power systems using wavelet analysis in the IEC standard waveform," Electric Power Systems Research, vol. 74, no. 4, pp. 200-208, 2006.

[9] IEEE Recommended Practice for Monitoring Electric Power Quality, IEEE Std 1159-1995, Institute of Electrical and Electronics Engineers, USA, 1995, pp. $11-25$.

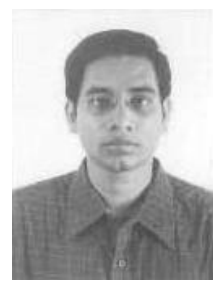

Debani Prasad Mishra received his B.E. (Electrical) degree from O.S.M.E., Keonjhar under BPUT, Odisha in 2006 and M. Tech (Power system) Degree from IIT, Delhi in 2010. Currently he is working at IIIT, Bhubaneswar as Assistant Professor in department of EEE. He worked as an Assistant Professor in KIIT University, Bhubaneswar from 2010-2011. He worked in ACC Cement captive power plant, Tikalria, U.P., from 2006 to 2008 